\title{
Discovery of Phenylglycine Lactams as Potent Neutral Factor Vlla Inhibitors
}

\author{
Nicholas R. Wurtz, ${ }^{* \dagger}$ Brandon L. Parkhurst, ${ }^{\dagger}$ Wen Jiang, ${ }^{\dagger}$ Indawati DeLucca, ${ }^{\dagger}$ Xiaojun Zhang, $^{\dagger}$ \\ Vladimir Ladziata, ${ }^{\dagger}$ Daniel L. Cheney, ${ }^{\dagger}$ Jeffrey R. Bozarth, ${ }^{\ddagger}$ Alan R. Rendina, ${ }^{\dagger}$ Anzhi Wei, ${ }^{\ddagger}$ \\ Joseph M. Luettgen, ${ }^{\ddagger}$ Yiming Wu, ${ }^{\ddagger}$ Pancras C. Wong, ${ }^{\ddagger}$ Dietmar A. Seiffert, ${ }^{\ddagger}$ Ruth R. Wexler, ${ }^{\dagger}$ \\ and E. Scott Priestley ${ }^{\dagger}$ \\ ${ }^{\dagger}$ Bristol-Myers Squibb R\&D, 350 Carter Road, Hopewell Township, New Jersey 08540, United States \\ ${ }^{\ddagger}$ Bristol-Myers Squibb R\&D, 311 Pennington Rocky Hill Road, Pennington, New Jersey 08534, United States
}

Supporting Information
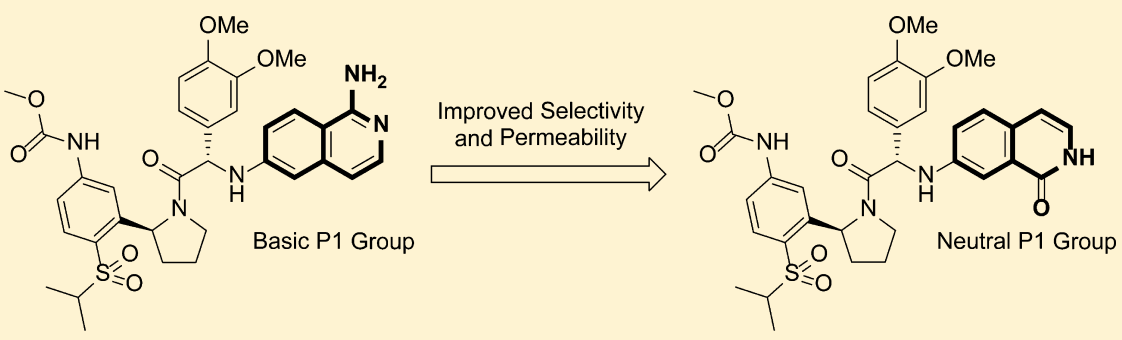

ABSTRACT: Inhibitors of Factor VIIa (FVIIa), a serine protease in the clotting cascade, have shown strong antithrombotic efficacy in preclinical thrombosis models with minimal bleeding liabilities. Discovery of potent, orally active FVIIa inhibitors has been largely unsuccessful because known chemotypes have required a highly basic group in the S1 binding pocket for high affinity. A recently reported fragment screening effort resulted in the discovery of a neutral heterocycle, 7-chloro-3,4dihydroisoquinolin-1 $(2 \mathrm{H})$-one, that binds in the S1 pocket of FVIIa and can be incorporated into a phenylglycine FVIIa inhibitor. Optimization of this P1 binding group led to the first series of neutral, permeable FVIIa inhibitors with low nanomolar potency.

KEYWORDS: TF-FVIIa inhibitor, anticoagulant, fragment-based drug design, neutral P1

$\mathrm{T}$ hromboembolic disorders remain a major cause of morbidity and mortality in the developed world. While anticoagulants such as the vitamin $\mathrm{K}$ antagonist, warfarin, and heparins have been the standard of care for many years, they possess significant limitations. Warfarin has highly variable effects in patients caused by significant drug and food interactions and therefore requires blood tests to maintain the desired therapeutic effect without excessive bleeding. ${ }^{1}$ Heparins require parenteral administration, which prevents their widespread use in long-term therapy. Several decades of research devoted to the discovery of new therapies have led to new oral anticoagulant drugs that target thrombin and FXa, with improved bleeding profiles. ${ }^{2}$ Despite overcoming many of the limitations of warfarin including having a wider therapeutic index, research to find safe, effective oral anticoagulants to complement existing therapies continues.

An early target in the clotting cascade that has attracted significant interest is the tissue factor-Factor VIIa complex (TFFVIIa). ${ }^{3,4}$ The complex initiates the extrinsic pathway in the clotting cascade by activating Factors X and IX. Studies show that inhibition of the TF-FVIIa complex by a small molecule in preclinical models demonstrates strong antithrombotic efficacy and a wide therapeutic index with respect to bleeding time. ${ }^{5}$
Inhibition of the TF-FVIIa complex may also have therapeutic potential in other areas such as cancer or inflammation due both to its antithrombotic effect and to other cellular signaling pathways. ${ }^{6}$ A parenterally dosed FVIIa inhibitor has prevented tumor growth in mouse models and was in clinical trials for treatment of pancreatic cancer. ${ }^{7,8}$

Despite years of study, the discovery of a potent, orally available FVIIa inhibitor remains a challenge to the field. FVIIa, like other trypsin-like serine proteases, has a deep S1 pocket ${ }^{9}$ with Asp189 positioned at one end. The adjacent 190 position contains a serine, which makes the FVIIa S1 pocket smaller and more hydrophilic than other serine proteases such as FXa, and thrombin, which have alanine at this position. ${ }^{10}$ The majority of reported FVIIa inhibitors contain a highly basic moiety such as an amidine $e^{4,11}$ that forms a bidentate salt-bridge with Asp189 for high affinity binding. Optimizing these structural classes into orally available inhibitors is difficult without use of prodrugs because amidines and other highly basic groups exist as charged species at physiological $\mathrm{pH}$ (7.4) and lack

Received: July 22, 2016

Accepted: September 16, 2016

Published: September 17, 2016 
permeability. The basic P1 groups can also preclude selectivity over other serine proteases, which have Asp189 and similar S1 pockets. Many of these shortcomings can be overcome with weakly basic P1 groups such as aminoisoquinoline and pyrrolopyridine. ${ }^{12-16}$ Furthermore, examples from the discovery of oral FXa inhibitors demonstrate that there are advantages of moving toward neutral groups that bind in the P1 pocket. ${ }^{2}$

There have been a few reports of neutral P1 groups with modest FVIIa potency. One of the successful neutral P1 groups is the meta-benzamide. ${ }^{15,18}$ In addition to forming a hydrogen bond with Asp189, the primary amide makes two interactions with the backbone carbonyl of Gly219: A benzamide $\mathrm{N}-\mathrm{H}$ hydrogen bonds with the oxygen of the Gly219 carbonyl, while the carbonyl of the amide forms a dipolar interaction ${ }^{19}$ with the

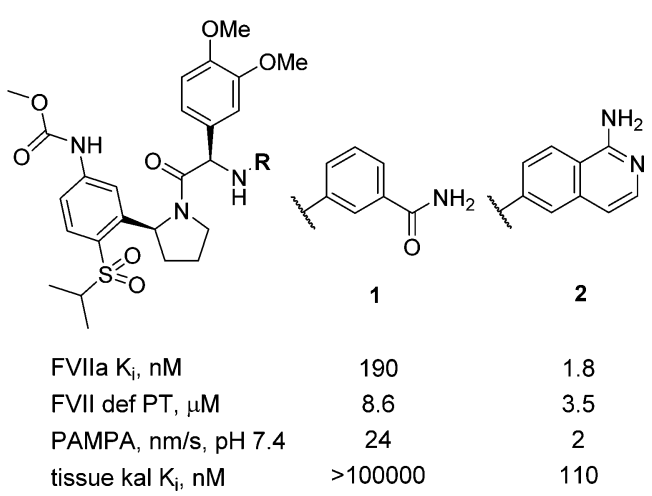

Figure 1. Comparison of previously discovered phenyl pyrrolidine FVIIa inhibitors with basic and neutral P1 groups.

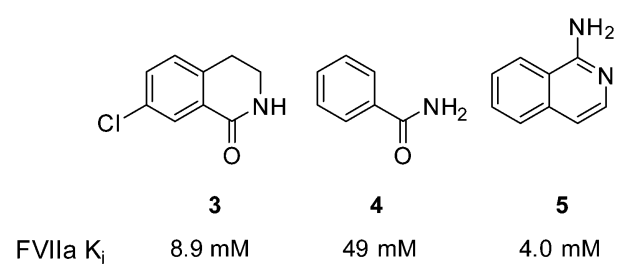

Figure 2. Fragments that bind in the $S 1$ pocket of FVIIa.

Scheme 1. General Synthesis of Phenylglycine Lactams ${ }^{a}$

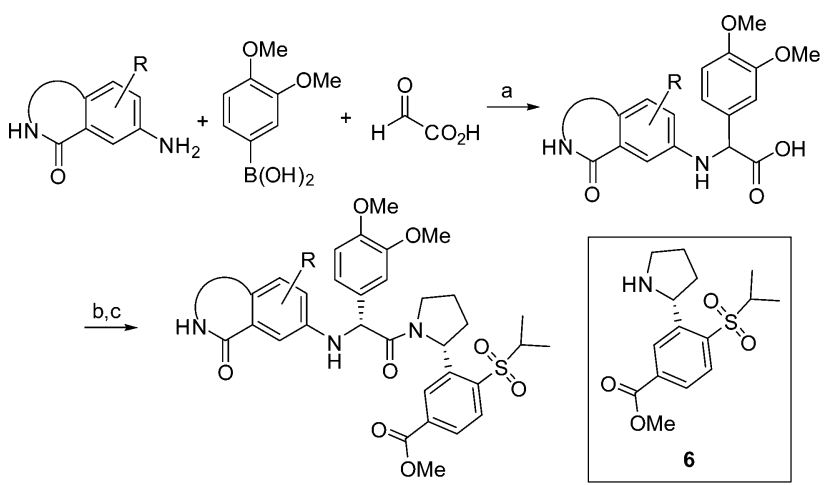

${ }^{a}$ Reagents and conditions: (a) acetonitrile/DMF (4:1), microwave, 100 C, 20-80\%; (b) EDCI, 6, DIEA, HOAt, DCM, 20-80\%; (c) HPLC separation.

carbon in the Gly219 carbonyl. In our phenylglycine series, ${ }^{16}$ we synthesized a library of substituted phenyl P1 groups and discovered that the only active compound was meta-benzamide 1, which provides a compound with modest potency, but good permeability and selectivity. Conversely, the corresponding aminoisoquinoline 2 (which is expected to be protonated under physiologic conditions) provides a potent inhibitor (FVIIa $K_{\mathrm{i}}=$ $1.8 \mathrm{nM}$ with insufficient permeability and low selectivity for enzymes such as tissue kallikrein, which promotes adverse cardiac remodeling when inhibited. ${ }^{20}$ Both compounds have good activity in the Factor VII deficient prothrombin time assay (FVII def PT), which measures the time to prolong blood coagulation by 2 -fold (Figure 1 ).

In order to search for a P1 that combined potency, permeability, and selectivity, we carried out a small molecule fragment screening effort, ${ }^{21}$ which involved a protocol starting with virtual screening, followed by NMR and enzymatic screening, and ending with crystallography. One high priority

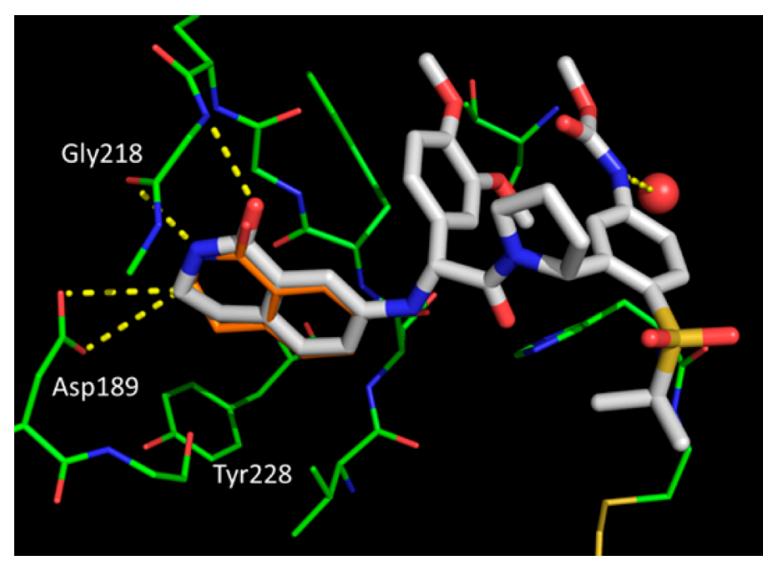

Figure 3. Crystal structures of compounds $\mathbf{3}$ (orange) and $\mathbf{8}$ (white) at 2.2 and $2.5 \AA$, respectively, bound in the active site of FVIIa. ${ }^{21}$ Crystal structures of compounds $\mathbf{3}$ and $\mathbf{8}$ bound in the active site of FVIIa. Only key residues in the $\mathrm{S} 1$ pocket are shown.

hit identified during our efforts was the dihydroisoquinolin$1(2 \mathrm{H})$-one 3 , which binds with a 5-fold higher affinity than benzamide 4 and is comparable in potency to 1 -aminoisoquinoline 5 (Figure 2). The structure led us to consider a similar binding orientation to the meta-benzamide P1. Initially this looked unlikely because the aliphatic region of the lactam overlapped with the same hydrophilic region where benzamidines form the charged bidentate hydrogen bond with Asp189. Herein we report our results for incorporation of this small molecule screening hit into the phenylglycine scaffold and subsequent optimization of the resulting FVIIa inhibitors.

In order to explore the various regions of the phenylglycine chemotype, a modular synthesis was pursued. Limited success in coupling aryl bromides to phenylglycine amines using various palladium- and copper- $\mathrm{N}$-arylation protocols led us to pursue another synthetic route. The Petasis three-component coupling $^{16,22}$ with the neutral P1 fragments, dimethoxyphenyl boronic acid, and glyoxylic acid (Scheme 1) yielded the phenylglycine scaffold. Neutral P1 fragments were synthesized or purchased as the corresponding aniline (see Supporting Information). Pyrrolidine $\mathbf{6}$ was coupled to the resulting phenylglycine carboxylic acids and the diastereomers were separated using preparatory HPLC to give the target compounds. The stereochemistry of the active diastereomer was assigned based on crystal structures of related compounds bound to FVIIa (not shown). While this chemistry sequence did not allow introduction of the P1 groups in the final synthetic step, the two step sequence provided a robust route 
Table 1. Activity and Permeability of FVIIa Inhibitors with Neutral P1 Substituents<smiles>CCCN[C@@H](C(=O)N1CCC[C@H]1c1cc(NC(=O)OC)ccc1S(=O)(=O)C(C)C)c1ccc(OC)c(OC)c1</smiles><smiles>[Z4]C(C)c1ccc2c(=O)[nH][nH]c(=O)c2c1</smiles>

to access all the desired compounds. The route also allowed facile SAR exploration via alteration of the boronic acid and phenylpyrrolidine components in the reaction sequence.

As reported previously, ${ }^{21}$ the 3,4-dihydroisoquinolin-1(2H)one was spliced into the phenylglycine FVIIa inhibitor series, producing compound $\mathbf{8}$ with similar potency in the FVIIa binding assay and increased permeability relative to the corresponding P1 meta-benzamide 1. Overlaying the crystal structures of the fragment 3 and phenylglycine 8 shows a close overlay of the dihydroisoquinolinone atoms in the S1 binding pocket (Figure 3). Both structures maintain the interactions with the Gly218 observed with the meta-benzamide. Further optimization of the P1 group is shown in Table 1. The open form of the lactam, methyl benzamide 9, is inactive, demonstrating the importance of tying back the lactam to maintain key interactions with Gly218. Interestingly, the aliphatic region of the lactam is in approximate vDW contact
Table 2. Activity and Permeability of Substituted Isoquinolinones<smiles>CCCN[C@@H](C(=O)N1CCC[C@H]1c1cc(NC(=O)OC)ccc1S(=O)(=O)C(C)C)c1ccc(OC)c(OC)c1</smiles>

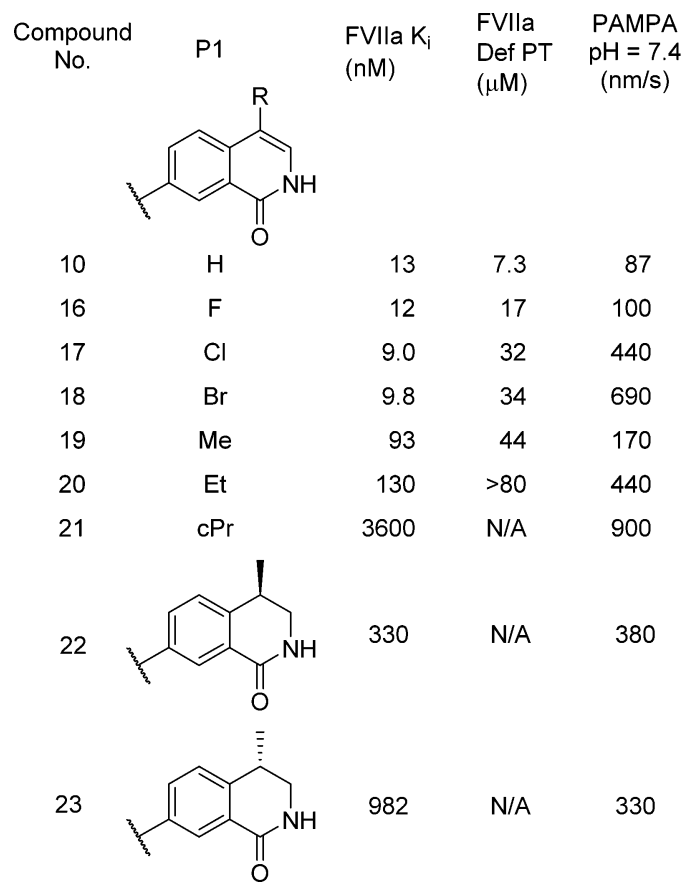

of the side chain of Asp189, placing the aliphatic moiety next to the polar acid, which is unprecedented to our knowledge.

To determine if an unsaturated ring would bind preferentially in the narrow pocket, isoquinolin-1 $(2 H)$-one 10 was synthesized, resulting in a 10 -fold potency increase in the binding assay and a 2 -fold increase in the clotting assay. Incorporation of nitrogen in the $\mathrm{C}-4$ position to produce quinazolin-4(3H)-one 11 maintained potency, but lost permeability. Moving the nitrogen to the three position in the phthalazin-1 $(2 \mathrm{H})$-one 12 resulted in a 60 -fold loss in potency, likely caused by the electrostatic repulsion with Asp189. The carbonyl in the C-4 position in 2,3-dihydrophthalazine-1,4-dione 13 recaptured some of the binding affinity but still only resulted in a compound with moderate potency. Like the isoquinolin-1 $(2 \mathrm{H})$-one, the flat isoindolin-1-one $\mathbf{1 4}$ produced a potent compound, albeit with little permeability. The introduction of the hydrogen bond donor in the $1 \mathrm{H}$ indazol-3 $(2 \mathrm{H})$-one $\mathbf{1 5}$ resulted in a loss of potency.

FVIIa crystal structures contain a conserved water molecule above the Tyr228 side chain. Since targeting this binding site and displacement of a similar water molecule by FXa inhibitors resulted in significant potency increase, we decided to target this region by substitution of the isoquinolin-1 $(2 \mathrm{H})$-one at the C-4 position. All C-4 halogenated analogues 16-18 gave equipotent inhibitors in the binding assay with the expected trend of increasing permeability with the size of the halides (Table 2). The clotting assay potency decreased for the $\mathrm{Cl}$ and 
Table 3. Activity and Permeability of Substituted Isoindolinones

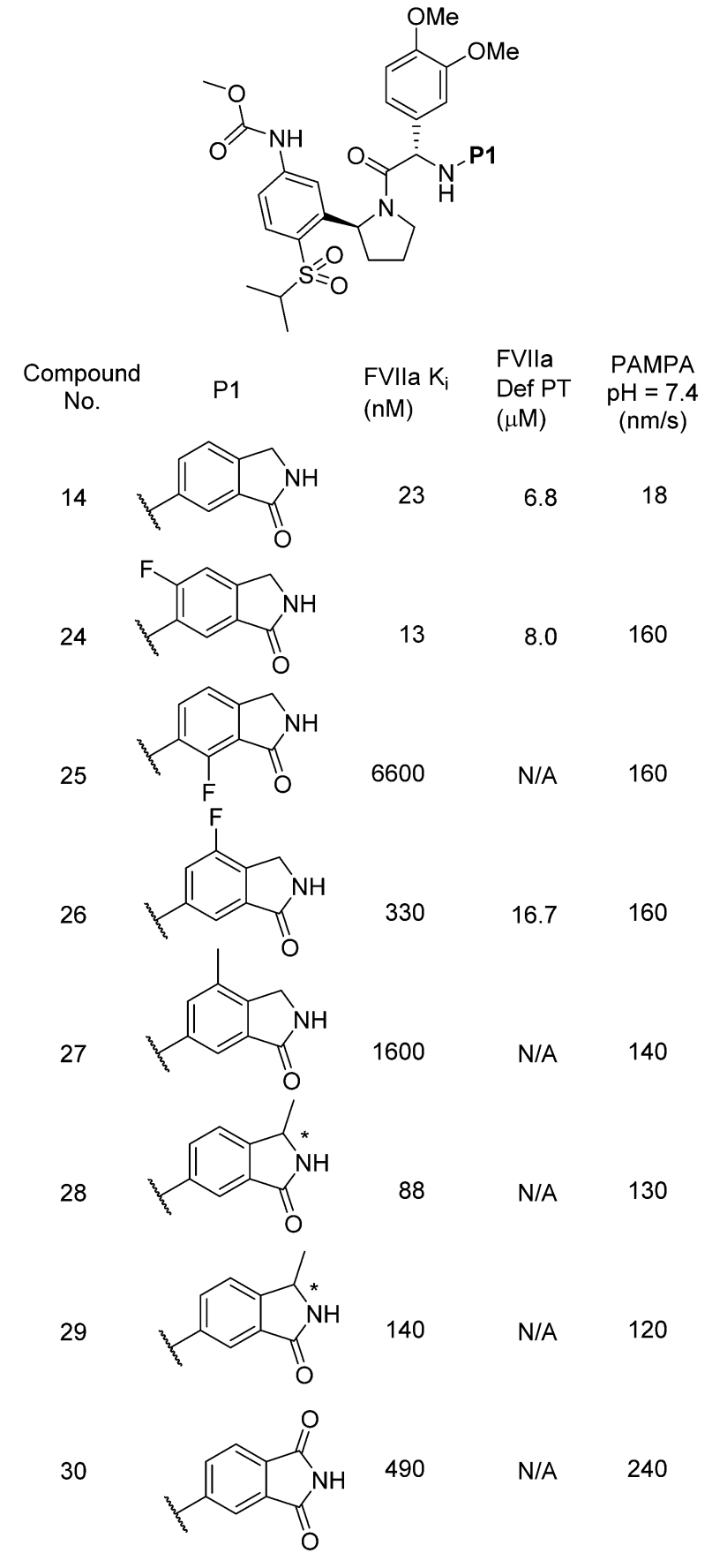

$\mathrm{Br}$, likely due to increased protein binding. Methyl and ethyl substitution at the C-4 position in 19 and 20 resulted in $\sim 10 X$ loss in potency, while cyclopropyl analogue $\mathbf{2 1}$ resulted in $\sim 300$-fold loss in potency. The difference in potency between the halo and alkyl substituted series may be due to differential desolvation effects and the favorable electrostatic interactions of the aryl halides with the S1 pocket, a subject of current investigation. Methylation of the 3,4-dihydroisoquinolin$1(2 \mathrm{H})$-ones at a similar position, compounds 22 and 23, resulted in moderate losses in potency. While the expected increase in potency for the Tyr228 interaction was not observed, the increase in permeability arising from halogenation

$\begin{array}{lcc}\text { Compound } & \mathbf{1 0} & \mathbf{2 4} \\ \text { FVIla } \mathrm{K}_{\mathrm{i}}, \mathrm{nM} & 13 & 13 \\ \text { FVII def } \mathrm{PT}, \mu \mathrm{M} & 7.3 & 8.0 \\ \text { PAMPA, nm/s, pH 7.4 } & 87 & 160 \\ \text { Trypsin } \mathrm{K}_{\mathrm{i}}, \mathrm{nM} & >6000 & 2570 \\ \text { Plasmin } \mathrm{K}_{\mathrm{i}}, \mathrm{nM} & >27000 & 3400 \\ & & \\ \text { tissue kallikrein, FIXa, } & \text { All } & \text { All } \\ \begin{array}{l}\text { FXa, FXla, thrombin, } \\ \text { plasma kallikrein, TPA, }\end{array} & >10000 & >10000 \\ \text { Urokinase, aPC } & & \end{array}$

Figure 4. Potency, permeability, and selectivity of compounds $\mathbf{1 0}$ and 24 for other serine proteases. $K_{\mathrm{i}}$ values were averaged from two experiments. TF/FVIIa assays were performed with recombinant human enzyme, while other enzyme assays were performed with purified human enzymes. All assays were run at $25{ }^{\circ} \mathrm{C}$ except tissue kallikrein 1 , which was run at $37^{\circ} \mathrm{C}$. Detailed descriptions of the enzyme assay protocols have been reported. ${ }^{5}$

of the isoquinolin-1 $(2 \mathrm{H})$-one may be a useful discovery in the design of FVIIa P1 groups.

Substitution around isoindolin-1-one 14 was largely not tolerated. The exception was fluorination of the C-2 position, which resulted in equipotent compound $\mathbf{2 4}$ with significantly increased permeability (Table 3). However, fluorination of the C- 6 and C-3 position caused a 300-fold and $~ 30$-fold decrease in binding affinity in compounds 25 and 26, respectively. Methyl substitution at the C-3 position to give 27 proved to be 5-fold less potent than fluorine. C-8 methyl substitution on the lactam ring with either chirality resulted in more permeable compounds but a 4-6-fold loss in potency. A carbonyl at the same position in compound $\mathbf{3 0}$ resulted in an even larger loss of potency.

Overall, isoquinolin-1(2H)-one 10 and 2-fluoro isoindolinone $\mathbf{2 4}$ provided the best balance of in vitro permeability and potency in the phenylglycine series. A broader look at binding to other relevant serine proteases for these two compounds (Figure 4) shows excellent selectivity (>200-fold) over all other serine proteases tested, which is representative of the selectivity observed for all compounds in this series with low nanomolar potency. Both compounds had high clearance when dosed IV in dog, so they were not advanced into further studies (See Supporting Information).

A dihydroisoquinolin-1 $(2 \mathrm{H})$-one that binds in the $\mathrm{S} 1$ pocket of FVIIa was identified in a fragment screen and spliced into a phenylglycine FVIIa inhibitor series to produce a neutral, potent, selective, and permeable FVIIa inhibitor. Subsequent optimization of the lactam P1 group with more planar heterocycles that maintained the amide-protein interactions led to nanomolar inhibitors of FVIIa with an array of neutral P1 binding groups, which represents the first reported series of potent neutral Factor VIIa inhibitors. This collection of P1 binding groups creates a toolbox of neutral P1 groups that will allow modulation of molecular properties, such as permeability, clotting activity, and selectivity while maintaining potency, and should be broadly useful in the design of orally active FVIIa inhibitors. 


\section{ASSOCIATED CONTENT}

\section{S Supporting Information}

The Supporting Information is available free of charge on the ACS Publications website at DOI: 10.1021/acsmedchemlett.6b00282.

Syntheses and characterization data for new compounds (PDF)

\section{AUTHOR INFORMATION}

\section{Corresponding Author}

*(N.R.W.) Tel: 609-466-5099. E-mail: nicholas.wurtz@bms. com.

\section{Notes}

The authors declare no competing financial interest.

\section{ACKNOWLEDGMENTS}

We would like to thank Atsu Apedo for carrying out separations of final molecules.

\section{ABBREVIATIONS}

TF-FVIIa, tissue factor-factor VIIa; FVII def PT, factor VII deficient prothrombin assay; FIXa, factor IXa; FXa, factor Xa; FXIa, factor XIa; FXIIa, factor XIIa; aPC, active protein C; tissue kal, human tissue kallikrein 1; TPA, tissue plasminogen activator; EDCI, 1-ethyl-3-(3-diemethylaminopropyl)carbodiimide; HOAt, 1-hydroxy-7-azabenzotriazole

\section{REFERENCES}

(1) Ansell, J.; Hirsh, J.; Hylek, E.; Jacobson, A.; Crowther, M.; Palareti, G. Pharmacology and management of the vitamin K antagonists. Chest 2008, 133, 160S-198S.

(2) Pinto, D. J.; Smallheer, J. M.; Cheney, D. L.; Knabb, R. M.; Wexler, R. R. Factor Xa inhibitors: next-generation antithrombotic agents. J. Med. Chem. 2010, 53 (17), 6243-74.

(3) Girard, T. J.; Nicholson, N. S. The role of tissue factor/factor VIIa in the pathophysiology of acute thrombotic formation. Curr. Opin. Pharmacol. 2001, 1 (2), 159-163.

(4) Priestley, E. S. Tissue factor-fVIIa inhibition: update on an unfinished quest for a novel oral antithrombotic. Drug Discovery Today 2014, 19 (9), 1440-4.

(5) Wong, P. C.; Luettgen, J. M.; Rendina, A. R.; Kettner, C. A.; Xin, B.; Knabb, R. M.; Wexler, R.; Priestley, E. S. BMS-593214, an active site-directed factor VIIa inhibitor: enzyme kinetics, antithrombotic and antihaemostatic studies. Thromb. Haemostasis 2010, 104 (2), 261-9.

(6) Rao, L. V.; Pendurthi, U. R. Tissue factor-factor VIIa signaling. Arterioscler., Thromb., Vasc. Biol. 2005, 25 (1), 47-56.

(7) Prescott, J.; Thiemann, P.; Loury, D. PCI-27483, a small molecule inhibitor of Factor VIIa, inhibits growth of BxPC3 pancreatic adenocarcinoma xenograft tumors. Cancer Res. 2008, 68, 5669.

(8) Young, W. B.; Mordenti, J.; Torkelson, S.; Shrader, W. D.; Kolesnikov, A.; Rai, R.; Liu, L.; Hu, H.; Leahy, E. M.; Green, M. J.; Sprengeler, P. A.; Katz, B. A.; Yu, C.; Janc, J. W.; Elrod, K. C.; Marzec, U. M.; Hanson, S. R. Factor VIIa inhibitors: chemical optimization, preclinical pharmacokinetics, pharmacodynamics, and efficacy in an arterial baboon thrombosis model. Bioorg. Med. Chem. Lett. 2006, 16 (7), 2037-41.

(9) Schechter, I.; Berger, A. On the size of the active site in proteases. I. Papain. Biochem. Biophys. Res. Commun. 1967, 27 (2), 157-62.

(10) Banner, D. W.; D’Arcy, A.; Chene, C.; Winkler, F. K.; Guha, A.; Konigsberg, W. H.; Nemerson, Y.; Kirchhofer, D. The crystal structure of the complex of blood coagulation factor VIIa with soluble tissue factor. Nature 1996, 380 (6569), 41-6.

(11) Shirk, R. A.; Vlasuk, G. P. Inhibitors of Factor VIIa/tissue factor. Arterioscler., Thromb., Vasc. Biol. 2007, 27 (9), 1895-900.
(12) Miura, M.; Seki, N.; Koike, T.; Ishihara, T.; Niimi, T.; Hirayama, F.; Shigenaga, T.; Sakai-Moritani, Y.; Tagawa, A.; Kawasaki, T.; Sakamoto, S.; Okada, M.; Ohta, M.; Tsukamoto, S. Design, synthesis and biological activity of selective and orally available TF/FVIIa complex inhibitors containing non-amidine P1 ligands. Bioorg. Med. Chem. 2007, 15 (1), 160-73.

(13) Trujillo John, I.; Huang, H.-C.; Neumann William, L.; Mahoney Matthew, W.; Long, S.; Huang, W.; Garland Danny, J.; Kusturin, C.; Abbas, Z.; South Michael, S.; Reitz David, B. Design, synthesis, and biological evaluation of pyrazinones containing novel P1 needles as inhibitors of TF/VIIa. Bioorg. Med. Chem. Lett. 2007, 17 (16), 456874.

(14) Riggs, J. R.; Hu, H.; Kolesnikov, A.; Leahy, E. M.; Wesson, K. E.; Shrader, W. D.; Vijaykumar, D.; Wahl, T. A.; Tong, Z.; Sprengeler, P. A.; Green, M. J.; Yu, C.; Katz, B. A.; Sanford, E.; Nguyen, M.; Cabuslay, R.; Young, W. B. Novel 5-azaindole factor VIIa inhibitors. Bioorg. Med. Chem. Lett. 2006, 16 (12), 3197-200.

(15) Bolton, S. A.; Sutton, J. C.; Anumula, R.; Bisacchi, G. S.; Jacobson, B.; Slusarchyk, W. A.; Treuner, U. D.; Wu, S. C.; Zhao, G.; Pi, Z.; Sheriff, S.; Smirk, R. A.; Bisaha, S.; Cheney, D. L.; Wei, A.; Schumacher, W. A.; Hartl, K. S.; Liu, E.; Zahler, R.; Seiler, S. M. Discovery of nonbenzamidine factor VIIa inhibitors using a biaryl acid scaffold. Bioorg. Med. Chem. Lett. 2013, 23 (18), 5239-43.

(16) Zhang, X.; Jiang, W.; Jacutin-Porte, S.; Glunz, P. W.; Zou, Y.; Cheng, X.; Nirschl, A. H.; Wurtz, N. R.; Luettgen, J. M.; Rendina, A. R.; Luo, G.; Harper, T. M.; Wei, A.; Anumula, R.; Cheney, D. L.; Knabb, R. M.; Wong, P. C.; Wexler, R. R.; Priestley, E. S. Design and Synthesis of Phenylpyrrolidine Phenylglycinamides As Highly Potent and Selective TF-FVIIa Inhibitors. ACS Med. Chem. Lett. 2014, 5 (2), 188-92.

(17) Kansy, M.; Senner, F.; Gubernator, K. Physicochemical high throughput screening: parallel artificial membrane permeation assay in the description of passive absorption processes. J. Med. Chem. 1998, 41, 1007-1010.

(18) Miura, M.; Seki, N.; Koike, T.; Ishihara, T.; Niimi, T.; Hirayama, F.; Shigenaga, T.; Sakai-Moritani, Y.; Kawasaki, T.; Sakamoto, S.; Okada, M.; Ohta, M.; Tsukamoto, S. Potent and selective TF/FVIIa inhibitors containing a neutral P1 ligand. Bioorg. Med. Chem. 2006, 14 (23), 7688-705.

(19) Paulini, R.; Muller, K.; Diederich, F. Orthogonal multipolar interactions in structural chemistry and biology. Angew. Chem., Int. Ed. 2005, 44 (12), 1788-805.

(20) Pons, S.; Griol-Charhbili, V.; Heymes, C.; Formes, P.; Heudes, D.; Hagege, A.; Loyer, X.; Meneton, P.; Giudicelli, J.; Samuel, J.; Alhenc-Gelas, F.; Richer, C. Tissue kallikrein deficiency aggravates cardiac remodelling and decreases survival after myocardial infarction in mice. Eur. J. Heart Failure 2008, 10, 343-351.

(21) Cheney, D. L.; Bozarth, J. M.; Metzler, W. J.; Morin, P. E.; Mueller, L.; Newitt, J. A.; Nirschl, A. H.; Rendina, A. R.; Tamura, J. K.; Wei, A.; Wen, X.; Wurtz, N. R.; Seiffert, D. A.; Wexler, R. R.; Priestley, E. S. Discovery of novel P1 groups for coagulation factor VIIa inhibition using fragment-based screening. J. Med. Chem. 2015, 58 (6), 2799-808.

(22) Petasis, N. A.; Zavialov, I. A. A New and Practical Synthesis of alpha-Amino Acids from Alkenyl Boronic Acids. J. Am. Chem. Soc. 1997, 119, 445-446. 